\title{
Microbiological Spectrum of Brain Abscess at a Tertiary Care Hospital in South India: 24-Year Data and Review
}

\author{
V. Lakshmi, ${ }^{1}$ P. Umabala, ${ }^{1}$ K. Anuradha, ${ }^{1}$ K. Padmaja, ${ }^{1}$ C. Padmasree, ${ }^{1}$ \\ A. Rajesh, ${ }^{2}$ and A. K. Purohit ${ }^{2}$ \\ ${ }^{1}$ Department of Microbiology, Nizam's Institute of Medical Sciences, Panjagutta, Hyderabad, \\ Andhra Pradesh 500082, India \\ ${ }^{2}$ Department of Neurosurgery, Nizam's Institute of Medical Sciences, Panjagutta, Hyderabad, \\ Andhra Pradesh 500082, India \\ Correspondence should be addressed to V. Lakshmi, vemulakshmigorthi@gmail.com
}

Received 24 November 2010; Accepted 15 September 2011

Academic Editor: S. K. Shankar

Copyright ( 2011 V. Lakshmi et al. This is an open access article distributed under the Creative Commons Attribution License, which permits unrestricted use, distribution, and reproduction in any medium, provided the original work is properly cited.

\begin{abstract}
Intracranial abscesses are life-threatening infections that pose a diagnostic challenge not only to the neurosurgeon but also to the microbiologists. Detailed studies documenting the spectrum of infecting agents involved in brain abscesses are limited from India. Materials and Methods. This is a retrospective analysis of 352 samples from 1987 to 2010 analyzed at a tertiary care hospital in South India from 1987 to 2010, to document the changing trends with time. Results. The age of the patients ranged from 2 to 80 years, a larger number of males being affected. Otogenic infections were the most common cause while cryptogenic abscesses were $20 \%$. Gram stain and culture positivity were $78 \%$ each. Gram-positive and negative facultative aerobes and obligate anaerobes were also on the rise. Unusual organisms, like Burkholderia pseudomallei, Salmonella typhi, Nocardia species, Cladosporium bantiana, Fonsecaea pedrosoi, Entamoeba histolytica, and Acanthamoeba were also isolated and/or detected from the brain abscesses aspirate or resected tissue. Summary. New and emerging pathogens associated with brain abscess, especially in immunosuppressed individuals, have renewed the necessity of an early detection, and it will be of great value in appropriate management of patients with brain abscess.
\end{abstract}

\section{Introduction}

Intracranial abscesses (usually referred to as brain abscess), though uncommon in developed countries, are serious, lifethreatening infections [1-3]. Major advances, such as stereotactic neurosurgical procedures, discovery of newer antibiotics, especially metronidazole against anaerobes and ceftriaxone which effectively crosses the blood brain barrier, and newer imaging techniques for early detection of brain abscesses [3], have lead to a substantial reduction in the mortality $[4,5]$. Despite these advances, brain abscess remains a potentially fatal central nervous system (CNS) disease, especially in developing countries [3-9].

Difficulties in the diagnosis of intracranial abscess are mainly due to protean clinical manifestations and similarities in the imaging and morphologic appearance of some intracranial mass lesions, like cystic gliomas and metastases.
The frequent delay in making the diagnosis renders this condition a significant challenge for the neurosurgeon [8$10]$ in the management of the case.

New and emerging pathogens, especially in immunosuppressed individuals, have renewed concern about the diagnosis and treatment of brain abscess and equally pose a challenge to the clinical microbiologist $[1,3,7,11]$. Meticulous microbiological investigations, including critical microscopic examination for all possible infectious agents and employing detailed microbial investigative armamentarium for the isolation of the organism(s) from the abscess material and from the probable primary site of origin of the infection elsewhere in the body, will definitely aid in the identification of the etiological agent(s) [2, 9-11]. These findings will enable the neurosurgeon and the infectious disease (ID) specialist to treat the brain abscess more rationally and appropriately $[5,7,8,10]$. 
It is well worth stating that a brain abscess is not only a neurosurgical emergency but also a microbiological emergency and a diagnostic challenge to both the disciplines [1, $10,12]$.

The objectives of this retrospective study were to analyze the microbiological findings in the purulent aspirates and/or tissue obtained from the brain abscesses and discuss the changing and evolving spectrum of infectious agents observed over the past 24 years with a brief review of other studies.

\section{Materials and Methods}

This is a hospital-based retrospective microbiological analysis of 352 brain abscess materials (purulent aspirates and/or tissue) that were received between 1987 and 2010, by the Microbiology Laboratory, at the Nizam's Institute of Medical Sciences, a tertiary care and a teaching hospital, in South India. The data was analyzed in two groups-group I, between 1987 and 1993 (published data) [12] and group II between 1994 and 2010, to document the changing trends in the microbial flora and treatment strategies.

(1) The demographic and clinical information of the patients was retrieved from the medical records section. Relevant data recorded included the age and sex of the patients, intracranial location of the abscess(es), the probable primary source of the infectious agents leading to the formation of the abscess(es).

(2) The microbiology data was retrieved from the microbiology records maintained as a database in the Microbiology Department.

(3) Wherever the resected brain abscess tissue was received, the histopathological features were corroborated to complement the culture results.

\section{Microbiological Investigations}

Specimen collected from a brain abscess (either through a burr hole or craniotomy) during any time of the day and submitted for microbiological investigations was considered as an emergency specimen and was processed immediately in the microbiology laboratory, on priority.

Specimen received between the years 1987 and 1993 (study group I) [12] was processed only for bacteria (aerobic and anaerobic), by routine microbiological procedures [13].

(i) Gram's stain was carried out on all specimens for bacteria, while Zeihl Neelsen's (ZN) stain for acid fast bacilli (AFB) was done in only one case of tuberculous brain abscess.

(ii) Aerobic cultures were performed on 7\% sheep blood agar and McConkey agar and incubated at $37^{\circ} \mathrm{C}$ for 48 hours, before being declared as sterile. All positive cultures were further processed for identification and antibiotic susceptibility patterns [13].

(iii) Anaerobic culture was performed on 7\% sheep blood agar plates and incubated in the Dynamicro Gaspak system. Metronidazole disc ( $5 \mu \mathrm{g})$ was placed to observe for antibiotic susceptibility of anaerobes. Isolates susceptible to metronidazole were considered to be anaerobes. Gram's stain of such isolates was carried out to confirm the morphology and the genus of the isolate. Aerotolerance test was performed to demonstrate that these isolates were obligate anaerobes.

(iv) The specimen from the tuberculous abscess showed AFB on $\mathrm{ZN}$ stain. The material was inoculated on Lowenstein Jensen's (LJ) medium, and the isolate was identified as Mycobacterium tuberculosis (M.tb) based on its rate of growth and susceptibility to para nitro benzoic acid [12].

The rest of the 302 brain abscess specimens (study group II) were processed for detection of bacteria (aerobes and anaerobes), mycobacteria, fungal pathogens including Nocardia and Actinomycetes, and parasites, by standard microbiological procedures [13] and by semiautomated identification systems (Mini API and the Vitek 2, bioMérieux, USA). Various detection procedures used were as per standard guidelines [13]. The anaerobic isolates were identified up to the genus level only.

\section{Results}

The average number of brain abscess specimens received for microbiological analysis was 21 per year.

Brain abscesses were diagnosed in all decades of life, with the ages ranging from 2 years to 80 years (mean of $28.5 \pm$ 17.6) and a male preponderance (male to female ratio was $2.7: 1$ ). The youngest patient (2 years) developed a frontal lobe abscess with an underlying septicemia and was referred to our institute for further management. The oldest patient was an 80-year-old lady, with chronic suppurative otitis media (CSOM) and a parietal lobe abscess.

Table 1(a) shows a comparison between the two study groups in terms of the location and source of infection of the brain abscess. Tables 1(b) and 1(c) highlight the topographic distribution of the abscesses and the probable source of infection documented in study group II.

There were $310(88.1 \%)$ nontraumatic and $42(11.9 \%)$ posttraumatic brain abscesses in our study. Though solitary abscesses $(313 / 352 ; 88.9 \%)$ were more common, multiple abscesses $(39 / 302 ; 12.9 \%)$, predominantly otogenic, were noted only in the study group II. There were 6 cases of subdural empyema and 3 cases of extradural abscesses. The rest of the brain abscesses were intracranial, involving the brain parenchyma. The majority of these intracerebral abscesses were located in the parietal region 102/352 (28.9\%), followed by the frontal and the temporal lobes $(21 \%)$.

\section{Microbial Spectrum}

Staining and microscopy of the brain abscess material (pus and/or tissue) revealed the pathogens in 41/50 (82\%) [12] and $221 / 302(73.1 \%)$ of the cases in the 2 groups, respectively. In the rest of the cases, only polymorphonuclear cells 
TABLE 1: (a) Neuroanatomical location and source of infection of brain abscess comparison between the two study groups. (b) Neuroanatomical location and source of infection of solitary brain abscess in study group II $(n=263)$. (c) Location and source of infection of multiple brain abscesses in study group II $(n=39)$.

(a)

\begin{tabular}{|c|c|c|c|c|c|c|c|c|c|c|c|c|}
\hline \multicolumn{13}{|c|}{ Primary source of infection } \\
\hline Location & \multicolumn{2}{|c|}{ CSOM } & \multicolumn{2}{|c|}{$\mathrm{CHD}$} & \multicolumn{2}{|c|}{ Pulm } & \multicolumn{2}{|c|}{ Trauma } & \multicolumn{2}{|c|}{ Cryptogenic } & \multicolumn{2}{|c|}{ Total } \\
\hline Group & $\mathrm{I}$ & II & I & II & I & II & I & II & $\mathrm{I}$ & II & $\mathrm{I}$ & II \\
\hline Parietal & 8 & 4 & 4 & 11 & 4 & 1 & 1 & 23 & 5 & 23 & 22 & 62 \\
\hline Frontal & 4 & 20 & 2 & 3 & - & 1 & - & 6 & 2 & 33 & 8 & 63 \\
\hline Temporal & 6 & 46 & 1 & 3 & - & - & - & 3 & 2 & 11 & 9 & 63 \\
\hline Cerebellar & 4 & 15 & - & - & - & 1 & - & - & - & 2 & 4 & 18 \\
\hline Occipital & 2 & 5 & 1 & 1 & - & - & - & - & 1 & 2 & 4 & 8 \\
\hline Subdural & - & 1 & 1 & - & 2 & - & - & - & - & - & 3 & 1 \\
\hline Total & 24 & 91 & 9 & 18 & 6 & 3 & 1 & 32 & 10 & 71 & 50 & 215 \\
\hline
\end{tabular}

CSOM: chronic suppurative otitis media, CHD: congenital heart disease, and Pulm: pulmonary.

Group I: 1987-1993 and Group II: 1994-2010.

(b)

\begin{tabular}{|c|c|c|c|c|c|c|c|c|c|c|c|c|}
\hline \multicolumn{13}{|c|}{ Primary source of infection } \\
\hline Location & ASOM & CSOM & PNS & $\mathrm{CHD}$ & Sepsis & Odont & Pulm & Trauma & Op trauma & Misc & Crypto & Total \\
\hline Parietal & - & 4 & - & 11 & 17 & 1 & 1 & 9 & 14 & 1 & 23 & 80 \\
\hline Frontal & - & 20 & 6 & 3 & 4 & - & 1 & 2 & 4 & 一 & 33 & 73 \\
\hline Temporal & - & 46 & 2 & 3 & 3 & 1 & 一 & 1 & 2 & 3 & 11 & 72 \\
\hline Cerebellar & 1 & 15 & - & - & 1 & - & 1 & - & - & 2 & 2 & 22 \\
\hline Occipital & - & 5 & - & 1 & - & - & - & - & - & - & 2 & 8 \\
\hline Subdural & - & 1 & - & - & 2 & - & - & - & - & - & - & 3 \\
\hline Epidural & - & - & - & - & 3 & - & - & - & - & - & - & 3 \\
\hline Thalamus & - & - & - & - & - & - & - & 1 & - & - & - & 1 \\
\hline Medullary & - & - & - & 一 & 一 & - & - & - & - & 一 & 1 & 1 \\
\hline Total & 1 & 91 & 8 & 18 & 30 & 2 & 3 & 13 & 20 & 6 & 71 & 263 \\
\hline
\end{tabular}

ASOM: acute suppurative otitis media, CSOM: chronic suppurative otitis media, PNS: paranasal sinusitis, CHD: congenital heart disease, Odont: odontogenic, Pulm: pulmonary, Op trauma: operative trauma, Misc: miscellaneous, and Crypto: cryptogenic.

E. hystolytica and Acanthamoeba cases not included in analysis.

(c)

\begin{tabular}{|c|c|c|c|c|c|c|c|c|c|c|c|c|}
\hline Location & ASOM & CSOM & PNS & $\mathrm{CHD}$ & Sepsis & Odont & Pulm & Trauma & Op trauma & Misc & Crypto & Total \\
\hline FP & - & 5 & - & 1 & 1 & - & - & 3 & - & - & - & 10 \\
\hline FT & - & 2 & - & 1 & - & - & - & 1 & - & - & - & 4 \\
\hline $\mathrm{FO}+\mathrm{SD}$ & - & 1 & - & - & - & - & - & - & - & - & - & 1 \\
\hline $\mathrm{F}+\mathrm{SD}$ & - & - & - & - & 1 & - & - & - & - & - & - & 1 \\
\hline $\mathrm{PO}$ & - & 5 & - & 3 & - & - & - & 1 & - & 3 & - & 12 \\
\hline $\mathrm{TP}$ & - & 5 & - & - & 1 & - & - & 3 & - & - & 1 & 10 \\
\hline $\mathrm{T}+\mathrm{SD}$ & - & 1 & - & - & - & - & - & - & - & - & - & 1 \\
\hline TOTAL & - & 19 & - & 5 & 3 & - & - & 8 & - & 3 & 1 & 39 \\
\hline
\end{tabular}

CSOM: chronic suppurative otitis media, PNS: paranasal sinusitis, CHD: congenital heart disease, Odont: odontogenic, Pulm: pulmonary, Op trauma: operative trauma, Misc: miscellaneous, Crypto: cryptogenic, FP: frontoparietal, FT: frontotemporal, FO: fronto-occipital, SD: subdural, F: frontal, PO: parieto-occipital, TP: temperoparietal, and T: temporal.

were seen, indicating an inflammatory process. Microscopy was positive on all the fungal, mycobacterial, Nocardial, and parasitic abscesses. However, the microscopy was negative in 12 bacterial culture-positive cases in group II.

Microbiological cultures for bacteria, mycobacteria, and fungi (included in the study group II only) revealed the etiological agent(s) in 44/50 (88\%) [12] and 203/302 (67.2\%) cases in the two groups. The spectrum of organisms isolated from the brain abscesses in both of the groups is compared in Tables 2(a), 2(b), and 2(c).

Gram-positive facultative aerobes were isolated more frequently than the gram-negative aerobes. Among the isolates, $35 / 44(79.5 \%)$ and $158 / 203(77.83 \%)$ were facultative aerobes, $7 / 44(15.9 \%)$ and 32/203 (15.8\%) were obligate 
TABLE 2: (a) Single and polymicrobial aerobic bacterial isolates from brain abscess. (b) Single and polymicrobial anaerobic isolates from brain abscess. (c) Miscellaneous bacterial and fungal isolates from brain abscess.

(a)

\begin{tabular}{|c|c|c|}
\hline \multirow{2}{*}{ Type of organism } & \multicolumn{2}{|c|}{ No. of isolates } \\
\hline & Group I $(n=35)$ & Group II $(n=158)$ \\
\hline \multicolumn{3}{|l|}{ Gram-positive facultative aerobes } \\
\hline Staphylococcus aureus & 11 & 51 \\
\hline Enterococci species (avium, faecium, and faecalis) & - & 32 \\
\hline Beta haemolytic Streptococci & 5 & 17 \\
\hline Alpha haemolytic Streptococci & 3 & 5 \\
\hline Streptococcus pneumoniae & 1 & 3 \\
\hline Diphtheroids & - & 1 \\
\hline \multicolumn{3}{|l|}{ Gram-negative facultative aerobes } \\
\hline Escherichia coli & 1 & 9 \\
\hline Klebsiella pneumoniae & 2 & 10 \\
\hline Enterobacter species & 2 & 6 \\
\hline Proteus species & 5 & 1 \\
\hline Morganella morganii & - & 1 \\
\hline Citrobacter freundii & - & 2 \\
\hline Pseudomonas aeruginosa & - & 5 \\
\hline Acinetobacter species & - & 4 \\
\hline Salmonella typhi & - & 1 \\
\hline Burkholderia pseudomallei & - & 1 \\
\hline Neisseria meningitides & - & 1 \\
\hline Total & 30 & 150 \\
\hline \multicolumn{3}{|l|}{ Facultative aerobes-polymicrobial } \\
\hline Klebsiella + Proteus & 2 & - \\
\hline BH Streptococci + Proteus & 1 & - \\
\hline S. aureus + Proteus & 2 & - \\
\hline Pseudomonas + Klebsiella & - & 1 \\
\hline Klebsiella + E. coli & - & 1 \\
\hline Klebsiella + S. aureus & - & 1 \\
\hline Pseudomonas + S. aureus & - & 1 \\
\hline Klebsiella + Acinetobacter + Enterococci & - & 1 \\
\hline S. aureus + Acinetobacter & - & 1 \\
\hline Enterococci + Strep. pneumonia & - & 1 \\
\hline S. aureus + E. coli & 一 & 1 \\
\hline Total & 5 & 8 \\
\hline
\end{tabular}

(b)

Type of organism

No. of isolates

$1987-1993(n=7)$

$1994-2010(n=32)$

Gram-positive obligate anaerobes

Peptococcus species

- 3

Peptostreptococcus species

3

11

Gram-negative obligate anaerobes

Bacteroides fragilis

1

Veillonella

Total

$-$


(b) Continued.

\begin{tabular}{|c|c|c|}
\hline \multirow{2}{*}{ Type of organism } & \multicolumn{2}{|c|}{ No. of isolates } \\
\hline & $1987-1993(n=7)$ & $1994-2010(n=32)$ \\
\hline \multicolumn{3}{|l|}{ Mixed anaerobic isolates } \\
\hline Peptostreptococcus $+B$. fragilis & - & 2 \\
\hline Prevotella melaninogenicus + Peptostreptococcus & - & 1 \\
\hline Total & - & 3 \\
\hline \multicolumn{3}{|l|}{ Mixed aerobic and anaerobic isolates } \\
\hline Pseudomonas + B. fragilis + Peptostreptococcus & - & 2 \\
\hline Strep. pneumonia + Proteus $+B$. fragilis & 1 & - \\
\hline Pseudomonas $+B$. fragilis & - & 2 \\
\hline S. aureus + Peptostreptococcus & - & 1 \\
\hline Proteus + Peptostreptococcus & - & 2 \\
\hline S. aureus + B. fragilis & 1 & 1 \\
\hline BH Streptococci $+B$. fragilis & 1 & 1 \\
\hline Pseudomonas + Veillonella & - & 1 \\
\hline Veillonella + Providencia stuartii & 一 & 1 \\
\hline Total & 3 & 11 \\
\hline
\end{tabular}

(c)

\begin{tabular}{|c|c|c|}
\hline \multirow{2}{*}{ Type of organism } & \multicolumn{2}{|c|}{ No. of isolates } \\
\hline & $1987-1993(n=2)$ & $1994-2010(n=13)$ \\
\hline \multicolumn{3}{|c|}{ Mycobacterial and Nocardial isolates from brain abscess } \\
\hline Mycobacterium tuberculosis & 1 & 6 \\
\hline Mycobacterium fortuitum & 1 & - \\
\hline Nocardia brasiliensis & - & 1 \\
\hline Nocardia asteroides & - & 1 \\
\hline \multicolumn{3}{|l|}{ Fungal isolates from brain abscess } \\
\hline Nocardia asteroides + Aspergillus terreus & - & 1 \\
\hline Aspergillus flavus & - & 1 \\
\hline Aspergillus flavus + BHS & - & 1 \\
\hline Cladosporium bantiana & - & 1 \\
\hline M. $t b+$ Fonsecaea pedroso $i$ & - & 1 \\
\hline Total & 2 & 13 \\
\hline
\end{tabular}

E. histolytica and Acanthamoeba cases not included in analysis.

anaerobes, and $2 / 44(4.5 \%)$ and $7 / 203$ (3.4\%) were mycobacteria. Nocardia species $3 / 203(1.5 \%)$ and fungal isolates $5 / 203(2.5 \%)$ were seen only in group II, probably due to the extensive methods employed. The obligate anaerobes were isolated mainly from the brain abscesses originating from the ear, paranasal, and oral infections. Mixed aerobic and anaerobic abscesses were also frequently encountered. There were 30 microscopy-positive abscesses with very small Gram-positive cocci in pairs and or chains, on Gram's stain, resembling anaerobic cocci. However, no organisms could be recovered by any of the culture methods used from these specimens.

\subsection{Bacterial Isolates (Tables 2(a) and 2(b)).}

5.1.1. Facultative Aerobes (180 Single and 13 Mixed Aerobes, Total 193) (Table 2(a)). Staphylococcus aureus (S. aureus)
$(68 / 192,35.2 \%)$ was the most common isolate, often as a single isolate. Compared to the group I, the incidence of $S$. aureus infections was about 4 times more (Table 2(a)). These isolates were usually from brain abscesses associated with CSOM and trauma cases, in the study group II. There were 6/55 methicillin-resistant S. aureus (MRSA) isolates, from brain abscesses following a road traffic accident (RTA), and they were sensitive to vancomycin. The rest of the isolates were methicillin-sensitive S. aureus (MSSA) and sensitive to a broad range of antibiotics.

Enterococcus species $(34 / 193,17.6 \%)$ were isolated frequently from the cases with CSOM. Compared to the study group I, Enterococcus species appeared to be significant emerging pathogens of brain abscess at our centre. The species isolated were E. avium, E. faecalis, and E. faecium. All the isolates demonstrated a high susceptibility to betalactams and vancomycin. The high rates of isolation could 
be due to the use of chromogenic media for primary culture of the pus and automated identification systems like the Vitek 2.

Beta haemolytic Streptococci (22), alpha haemolytic Streptococci (8), and S. pneumoniae (5) were mostly otogenic and were highly susceptible to penicillin and other betalactams. The isolation of these important pathogens was more in the study group II, often as single isolates.

Aerobic Gram-negative bacilli $(62 / 193,32.1 \%)$ though mainly otogenic, these organisms were also frequently isolated from the postcraniotomy and hematogenic abscesses. The enteric bacilli, such as Escherichia coli (E. coli), Klebsiella pneumoniae (K. pneumoniae), Enterobacter species, Proteus species, Morganella morganii, and Providencia stuartii were the frequent isolates. Like the Gram-positive pathogens, the incidence of the Gram-negative bacilli, especially of multidrug-resistant and nosocomial pathogens, was seen to increase. E. coli and K. pneumoniae were highly resistant organisms producing extended-spectrum $\beta$-lactamase (ESBL). Nosocomial pathogens such as Pseudomonas aeruginosa (P. aeruginosa) and Acinetobacter baumannii (A. baumannii) were also isolated.

5.1.2. Obligate Anaerobes (22 Single and 17 Mixed, Total 39). An increasing incidence of polymicrobial anaerobic abscesses was documented. The spectrum of anaerobes in this study is shown in Table 2(b).

\subsubsection{Uncommon Gram-Negative Bacilli}

(i) B. pseudomallei (2 cases): One patient with melioidosis had presented with fever and abdominal pain and developed calvarial osteomyelitis and multiple parietal and sagittal sinus abscesses [14]. Another patient with a splenic abscess due to $B$. pseudomallei developed a frontal lobe brain abscess. However, the organism could not be isolated on culture from the brain abscess pus.

(ii) S. typhi was isolated from the brain abscess pus in a case of septicemia.

5.1.4. Mycobacterial Species (8/352, 2.2\%). All the 8 TB abscesses revealed AFB on microscopy, and $M$. $t b$ was isolated by culture on LJ Medium (study group I) and BACTEC 460 TB system (Becton Dickinson, USA). In our study group I, two cases of mycobacterial abscesses, $(M . t b$ (1) and $M$. fortuitum (1) [12] were documented, while there were 6 cases of tuberculous brain abscesses and one was a mixed fungal and TB abscess [15], in the study group II.

5.1.5. Nocardial Species (3/302, 1\%). There were 2 parietal lobe abscesses caused by $N$. asteroides in our group (one in a postrenal transplant patient and the other in a case of atopic dermatitis on steroids). We also reported a case of a young female with mycetoma on the back, who presented with an epidural abscess. $N$. brasiliensis was isolated from the sinuses on the skin and from the epidural abscess pus [16].
5.2. Fungal Agents. There were $5(1.7 \%)$ cases of fungal brain abscess in our series, that occurred in various clinical settings. The cases with Aspergillus abscesses had a fatal outcome.

We reported a case of a 23-year-old immunocompetent male who sustained a head injury and developed a capsuloganglionic region abscess due to the neurotropic fungus, Cladophialophora bantiana [17]. Diagnosis was made based on the microscopy, mycology, and histopathology findings of the aspirate obtained from the abscess. The patient responded clinically to amphotericin $B$.

\subsection{Protozoa}

(a) E. histolytica abscess: the trophozoites of E. histolytica were demonstrated on microscopy in a patient with cerebral amoebiasis. There was no evidence of diseases elsewhere. Patient had a complete recovery following a total excision of the abscess and Metronidazole therapy [18].

(b) A fatal case of an acute frontal lobe abscess due to Acanthamoeba, in an immunocompetent patient with meningoencephalitis, was documented. The cerebrospinal fluid (CSF) from the patient showed the Acanthamoeba (polygonal) cysts on Gram stain and calcofluor white stain. However, a coculture with $E$. coli was unsuccessful in isolating the organism [19].

\section{Discussion}

Brain abscesses have been well known and reported from the beginning of the Hippocratic era [10, 11]. Essentially, a brain abscess is a focal intraparenchymal collection of pus and is classified based on the anatomical location or the etiologic agent causing it. It begins as a localized area of cerebritis and evolves into a collection of pus surrounded by a vascularized capsule $[5,20]$.

Our data shows that the incidence of brain abscess continues to be significant in the neurosurgical clinical setting. The average incidence of brain abscess among all the spaceoccupying lesions in our institute is about $25 \%$. Recent studies from India have reported an average of 9 to 15 cases per year $[1,7,8]$, while studies from developed countries recorded a lower number, 5-12 cases per year [10, 21, 22]. As per our data and other studies, brain abscesses occur in all decades of life, with a male preponderance $[1,6,21,22]$. However, the reasons for the male preponderance, are not clear [1].

The spectrum of organisms seen in brain abscess usually depends on the primary source of infection. Gram-positive cocci, especially the Enterococci, and Gram-negative bacilli, especially E. coli and K. pneumoniae, were more frequently isolated in group II than in the earlier group I. This is probably due to the use of the Bact/alert system and direct inoculation of the purulent aspirates into the bottles. A larger number of antibiotic-resistant Gram-negative bacilli were isolated from cases in group II, probably due to the increased number of trauma cases in this group. 
6.1. Otogenic Abscesses. CSOM continues to be the most frequent predisposing condition in all age groups (24/50 (48\%) and $118 / 302(39 \%)$ in both the groups), respectively, time groups. Other studies from India also documented CSOM as a major source of brain abscess, $49 \%$ [1], 31.4\% [7], and $40 \%$ [8]. Though middle ear suppurative disease was seen to extend to temporal lobe or cerebellum $[1,23,24]$, multiple otogenic abscesses, often involving the frontal and parietal lobes, were seen in the study group II, probably due to arterial dissemination of infective emboli. Direct extension may also occur through osteomyelitis in the posterior wall of the frontal sinus, sphenoid, and ethmoid sinuses. This direct route of intracranial extension is more commonly associated with subacute and chronic otitic infection and mastoiditis than with sinusitis [4]. Frontal or ethmoid sinus infections generally spread to the frontal lobes. Odontogenic infections can spread to the intracranial space via direct extension or a route, generally to the frontal lobe [4].

All the otogenic abscesses, in our study, were of bacterial etiology (aerobic and/or anaerobic), with culture positivity of $85 \%$. Gram-positive facultative aerobes were the most frequent isolates from these abscesses, as also recorded in other studies $[1,6,7,23,24]$. An unusual case of concomitant TB (M. tb) and fungal (Fonsecaea pedrosoi) infections involving the middle ear cleft extending and destroying the craniovertebral junction at the skull base was successfully managed and treated at our centre [15]. Our study and others from India clearly suggest that middle ear infections need to be treated aggressively to reduce the incidence of brain abscesses $[1,24]$.

6.2. Odontogenic Abscesses. The oral and dental florae, mainly from the subgingival sites, are documented to be frequent sources of brain abscesses [25]. These sites are usually comprised of Streptococcus milleri and the Gram negative anaerobic bacilli. Two cases of odontogenic abscesses were noted in the study group II caused by S. aureus and Prevotella melaninogenica.

6.3. Cardiogenic Abscesses. Cyanotic congenital heart diseases such as tetralogy of Fallot (10/23, 43.5\%), transposition of the great vessels $(3 / 23,13 \%)$, and dextrocardia $(2 / 23,0.08 \%)$ are documented as risk factors for brain abscess $[1,8,26$, 27] and congenital pulmonary arteriovenous malformations [28]. We documented cardiogenic abscesses in 18\% (9/50) in the study group I [12] and 7.6\% (23/302) in the study group II. The age of the patients with cardiogenic abscess was less than 20 years though there was one 45 -year-old patient with an $S$. aureus abscess.

Patients with cyanotic heart disease have a right to left shunt of venous blood in the heart, bypassing the pulmonary circulation, thus leading to bacteremia, septicemia, and infective thromboembolism, usually in the brain. These patients also have low-perfusion areas in the brain due to chronic hypoxemia and secondary polycythemia. The abscesses, often multiple, occur at the junction of the gray and white matter. The parietal lobes are most commonly affected due to the large caliber and direct continuation of the middle cerebral artery [9]. The congenital arteriovenous anomalies become a nidus for the organisms, especially Gram-positive ones, spread to the brain leading to the development of brain abscess $[1,9]$. Nineteen of the $23(82.6 \%)$ cardiogenic abscesses in our series were positive for bacteria by microscopy and Gram-positive cocci, including facultative aerobes, and obligate anaerobes were isolated.

6.4. Traumatic Abscesses. Trauma to the skull, either following road traffic accident $(21 / 302 ; 7.2 \%)$ or a craniotomy $(20 / 302 ; 6.6 \%)$, is an important risk factor in the study group II, which was not encountered in the study group I, probably related to increase in speeding vehicles and availability of neurosurgical facilities. Thirty six of the forty one abscesses were solitary, and the parietal lobe was the most commonly affected site.

Microscopy revealed organisms in $92.7 \%$ abscesses, while 80.5 of them were culture positive. S. aureus infection was common in the RTAs, probably due to the direct implantation of the organism derived from the normal flora of the calvarial skin. On the other hand, the postcraniotomy abscesses had a spectrum that is predominantly of gramnegative facultative aerobes and was often a nosocomial infection. These isolates, most commonly E. coli and K. pneumoniae, had a high level of resistance to antibiotics. Strict aseptic measures during the postoperative management of the surgical wound and care of the intravascular catheters in these patients is very important and cannot be underscored $[6,11]$.

Postcraniotomy brain abscesses due to C. bantiana were reported by other centers [29-31]. The portal of entry of the fungus is either due to direct inoculation following trauma to the skull, either following RTA or craniotomy, inhalation of the spores, or a haematogenous spread to the brain [31].

6.5. Hematogenic Abscesses. Septicemia and/or sepsis elsewhere in the body is a probable cause for the spread of the organism from the primarily involved organ to the brain. We documented $10.9 \%$ hematogenic abscesses in the study group II. Twelve of the abscesses $(36.4 \%)$ had no detectable organisms on microscopy and were sterile on culture. Menon et al. recorded $40 \%$ sterile abscesses in patients on prior antibiotics [1].

Also calling metastatic abscesses from a remote site, the hematogenic abscesses are the commonest type in developed countries [3]. They are often multiple and typically occur at the junction of the white and gray matter, where the capillary blood flow is the slow [9]. They are more commonly seen along the distribution of the middle cerebral arteries and the parietal lobes, where the regional blood flow is the highest. The common systemic sources of infection are chronic pulmonary infections, skin pustules, bacterial endocarditis, and osteomyelitis. Those with a right to left vascular shunt as a result of congenital heart disease or pulmonary arteriovenous malformations are particularly susceptible. Being hematogenous, any lobe of the brain can be affected, and some of them could be multiple $[9,28]$. Though these abscesses are reported to contain a mixed flora [32], 
in the present series, the infected hematogenic brain abscesses were monomicrobial, since the septicemia is usually monomicrobial. Gram-negative pathogens were more frequently isolated from these abscesses, though unusual Gram-negative organisms such as S. typhi and B. pseudomallei were isolated from the hematogenic brain abscess pus.

6.6. Preexisting Pulmonary Lesions. Primary infections or secondarily infected pulmonary cavities can be a predisposing cause of brain abscess, especially in the immunocompromised patients [4]. Six cases of brain abscesses of a probable pulmonary origin were recorded in group I which include 1 M.tb, 1 M. fortuitum, and 4 bacterial abscesses [12]. In the study group II, there were 3 abscesses of proven pulmonary origin (organisms were isolated from the respiratory tract), which grew $N$. asteroides (a case of postrenal transplant) and M.tb (2 cases), respectively.

6.7. Urosepsis. The urinary tract infections are an important primary source of brain abscess. We documented an abscess in lower midbrain in a patient with urosepsis. The abscess was sterile, probably due to prior antibiotic therapy. A similar report of brain abscess due to urosepsis was reported in literature [33].

6.8. Intracranial and Meningeal Lesions. Brain abscess has been documented to occur following a contiguous spread from infected foci within the brain or meninges or a secondary infection of preexisting intracranial lesions $[4,33]$. Two brain abscesses from a primary infective focus within the brain or the meninges were documented in our study. One was a case of neurocysticercosis developing a temporal abscess due to Neisseria meningitides, probably a secondary infection of the cystic lesion. The other case was a case of pyogenic meningitis developing a frontoparietal abscess due to Peptostreptococcus species.

6.9. Osteomyelitis of the Calvarium. Osteomyelitis of the skull bones can be a primary focus with a contiguous spread to the underlying parenchyma [4] as noted in our study [14]. We documented an unusual case of B. pseudomallei calvarial osteomyelitis that had spread to both the parietal lobes and medially extended up to the sagittal sinus [14]. There were two other cases of calvarial osteomyelitis with sterile abscesses in the study group II.

6.10. Immunosuppression. Immunosuppression can predispose patients to the development of brain abscesses. With the increasing number of transplant surgeries, especially renal, in the recent times, it is a significant emerging problem [9]. Immunosuppression induced by steroids also may predispose the patients in developing brain abscess.

The spectrum of organisms noted in the immunosuppressed patients may not be found in immunocompetent individuals, and because of this, empirical therapy in these patients should be avoided [9]. Since, the imaging features of the abscess on computerized tomography or magnetic resonance imaging (MRI) also do not help in the diagnosis, attention should be directed to obtaining a microbiological diagnosis, whenever possible, so that appropriate antimicrobial therapy can be initiated without delay. The pus obtained from the abscess should be subjected to microbiological examination for fungal elements, AFB including Nocardia and parasites besides the routine aerobic and anaerobic cultures $[1,7,9]$. These compromised hosts with impaired T-lymphocyte or macrophage function are prone to develop infections with intracellular pathogens such as fungi (particularly Aspergillus species) and bacteria like Nocardia species $[6,11,34]$, especially emerging Nocardial species $(N$. cyriacigeorgica) [35].

Brain abscess was documented in 3 cases of renal transplantation. The aspirated materials on microscopy, and culture revealed growth of A. flavus, N. asteroids, and S. pneumoniae along with $S$. aureus in the 3 cases, respectively (Table 2(c)). Another fatal case of mycotic brain abscess in a case of atopic dermatitis on immunosuppressive therapy with steroids in a 5-year-old female child and multiple parietal abscesses secondary to A. terreus and $N$. asteroides, probably originating from the ears or the lungs, were noted. Dias et al. documented a case of Nocardial brain abscess in an elderly male with undetected diabetes mellitus [34, 36].

6.11. Cryptogenic Brain Abscesses. This group comprises of those abscesses where there would be no obviously demonstrable primary focus of infection elsewhere in the body nor any underlying predisposing condition leading to infection. The reported incidence of such cryptogenic abscesses, as per several studies ranges between 15 and 22\% [1, 10, 34]. The organisms in the cryptogenic abscesses have been shown to be derived from the upper respiratory tract and oral flora, comprising mainly of Streptococcal species and anaerobic $\operatorname{cocci}[1,10]$. In one of the studies, a patent foramen ovale was identified by echo cardiogram and was proposed as a possible way of migration and seeding of the oral flora to the brain [37].

We documented $23.3 \%$ brain abscesses as cryptogenic. Though the majority were solitary and sterile abscesses $(53 / 82,64.6 \%)$, the culture-positive cryptogenic abscesses $(19 / 82,26.4 \%)$ were caused mainly by a single Gram-positive facultative aerobe (12/19), among which S. aureus (MSSA) was the predominant isolate. The remaining cases were polymicrobial.

\section{Microscopy versus Culture}

The sensitivity of microscopy depends on the number of organisms in the specimen $\left(10^{3} \mathrm{CFU} / \mathrm{mL}\right)$. In our study, the Gram's stain was positive in 30 culture-negative cases, while it was negative in 12 culture-positive cases. There is a need to adopt methods to improve the detection rates especially by microscopy, which includes fluorescent staining using acridine orange (sensitivity $\left.\left(10^{2} \mathrm{CFU} / \mathrm{mL}\right)\right)$ [13], calcoflour white for fungal filaments, and by auramine rhodamine fluorescent stain for mycobacteria. 


\section{Culture for Various Organisms}

All the culture methods used in our study were to optimize the isolation of the various possible and cultivable etiologic agents. It has been shown that a direct inoculation and use of automated methods of culturing the pus, immediately after its aspiration, enhances the yield of organisms [7, 38]. Direct inoculation of the pus specimen into a standard anaerobic BacT/Alert bottle (bioMérieux, USA) facilitated early and better yield of the organisms. The unvented, bottled anaerobic medium facilitates the growth of both facultative aerobes and the obligate anaerobes, and the yield is further enhanced by the shaking incubator, inbuilt in the bacT/alert system [38].

Despite the meticulous conventional microbiological procedures, $6 / 50(12 \%)$ and 99/302 (32.8\%) abscesses were sterile, in our group, respectively. The reported incidence of sterile abscesses, from other centers, has ranged between $0 \%$ and $43 \%[1,7,39]$. The yield of organisms by culture also directly depends on the prior use of antibiotics [11]. This could be one of the reasons for an increased incidence of sterile abscesses in group II of our study.

Infections with $P$. aeruginosa and $A$. baumannii are caused by contiguous extension to brain and meninges from an ear, mastoid, paranasal sinus surgery, or diagnostic procedures. In some patients, the involvement of the CNS is due to spread of the organism from infective endocarditis, pneumonia, or urinary tract infection [3]. These organisms have emerged as important and highly resistant nosocomial pathogens in recent years.

Brain abscess due to unusual Gram-negative bacilli was observed in the study group II only, probably due to the improved microbiological diagnostic techniques and automated identification systems used.

(i) Salmonella typhi is a rare pathogen in brain abscess and probably spreads to the brain hematogenously [40].

(ii) B. pseudomallei (agent of Melioidosis): brain abscess is a complication of a neurological infection [11]. Isolation of $B$. pseudomallei from the specimen remains the "gold standard" in diagnosis. The microbiologist should be well versed with the colony morphology and the antibiogram of these important yet not very fastidious organisms. Ceftazidime remains the drug of choice in the early phase of treatment, followed by a prolonged therapy with cotrimoxazole [14].

Anaerobic brain abscesses: the earlier studies on brain abscesses from India were specifically on anaerobic infections [41-43]. The obligate anaerobes are significant pathogens of a brain abscess and often occur as mixed infection either with another anaerobe or a facultative aerobe, as seen in our group. They are generally associated with otogenic and odontogenic infections.

Anaerobes are highly susceptible to metronidazole [41]. Subsequent to its addition to the antibiotic treatment regimen of a brain abscess and widespread use, the incidence of anaerobic infections has declined [1, 12, 41]. Attention to proper anaerobic isolation techniques is essential for a good recovery $[11,13]$ as is evidenced in the increased yield of anaerobes in our study group II (Table 2(c)). Since conventional anaerobic cultures require 2-3 days of incubation, it is essential to use alternative techniques for their early detection $[44,45]$. Gas liquid chromatography (GLC) is a routine method used in the identification and differentiation of anaerobes from aerobes in clinical samples on the basis of the presence of volatile and nonvolatile fatty acids on the chromatogram. Computer-aided GLC is commercially available and is a means of rapid microbial identification $[44,45]$. However, the equipment and cost are the limiting factors for a routine use of GLC in resourcerestricted conditions.

Tuberculous brain abscess results when mycobacteria gain entry to the brain parenchyma, by a hematogenous route from a remote site, usually the lungs, unlike TB Meningitis, which occurs via lymphatic spread from cervical lymph nodes. The TB bacilli are immobilized in end arteries, which lead to formation of submeningeal tuberculous foci and either result in a tuberculoma or undergo a central caseation and liquefaction to form an abscess [46-49]. This phenomenon is very rare and commonly occurs in patients with cell-mediated immunity and is mostly focal and usually secondary to a primary focus in the lungs. Histologically and clinically, these abscesses are devoid of a granulomatous reaction, similar to pyogenic abscesses [19].

For appropriate therapy and clinical management, a TB abscess must be differentiated from a tuberculoma. The criteria for the diagnosis of a TB brain abscess, laid down by Whitener in 1978, should be fulfilled in the diagnosis of a TB brain abscess [50]. These include (i) evidence of a true abscess formation within the brain, as confirmed during surgery, (ii) histological proof of the presence of inflammatory cells in the abscess wall (histologically, the abscess walls are usually devoid of epitheloid and giant cells, unlike in a tuberculoma [13], and (iii) demonstration of AFB and isolation of M.tb from the abscess pus. Though not included in this data, we had reported a case of calvarial tuberculous osteomyelitic abscess, which was successfully managed [51].

Nocardial brain abscesses are rare and account for about $1-2 \%$ of all cerebral abscesses [52-54]. The entity is being increasingly reported in the present era of transplant surgeries and immunosuppressive therapies. The infection may occur as an isolated lesion or as a part of a disseminated infection of a pulmonary or a cutaneous infection $[6,11,16$, $34,52,53,55]$. Almost all the patients with Nocardial brain abscesses have a defective cell-mediated immunity [11]. An early detection and treatment are very important since the mortality is three times higher than that of other bacterial brain abscesses. Though N. asteroides and N. brasiliensis are the common species, other species are also being isolated from brain abscess [52]. The diagnosis of Nocardial brain abscess requires a high index of clinical suspicion, with an attempt for an early tissue and microbiological diagnosis [34].

Invasive fungal infections remain a life-threatening complication in children with hematological malignancies. The brain is a common site of haematogenously disseminated fungal infections from an extracranial focus [56]. Cerebral 
aspergillosis occurs in about 10 to $20 \%$ of all cases of invasive aspergillosis and has a very poor prognosis. The outcome depends on the early recognition of the causative organism and prompt initiation of antifungal treatment. Hence, every attempt should be made to detect fungal filaments in the brain abscess material submitted [11].

\section{Alternative and Advanced Techniques to Improve Detection of the Infecting Pathogen(s)}

Microscopy-positive but culture-negative abscesses, especially the bacterial, are not unusual and pose a challenge to the microbiologist(s). The organisms, often the nutritionally demanding Streptococcus species and the anaerobic cocci, generally will not be recovered on routine culture media. Also cultures will be negative when the number of organisms in the abscess pus is less. Such specimen should ideally be processed for the etiologic agent by more sensitive assays. At present, these highly sensitive and sophisticated assays are being increasingly used in advanced centers for research purposes.

(1) Broad range real-time polymerase chain reaction (RT PCR) using 16srRNA or DNA will help amplify and detect the probable bacterial nucleic acid, as shown by several studies [57-62]. A subsequent sequencing of the amplified product is performed to further improve the specificity. With the recent advances, the molecular methods are gradually making inroads into routine diagnostic laboratories and in the coming years may become important primary tools in the early detection of the etiologic agent. However, the specificity and the cost of these assays are important limiting factors [63] in the underdeveloped and developing countries.

(2) In vitro nuclear magnetic resonance (NMR) used for detecting the specific spectral pattern of the amino acids and other volatile substances released by metabolic processes of the bacteria has been applied as a means of differentiating abscesses from other spaceoccupying lesions in the brain [64-66]. However, the need for NMR with very high Tesla and an expert analysis and interpretation of the spectral patterns are the limiting factors.

(3) Gas liquid chromatography (GLC) on the pus sample is also an alternative method, especially for the anaerobic abscesses.

\section{Conclusions}

As evidenced by our data, the microbiology of intracranial abscesses is complex. The detection and identification of the causative pathogen(s) are the cornerstones of diagnosis for an appropriate management and therapeutic optimization. The value of a promptly evaluated microscopy coupled with meticulous and elaborate culture methods of the abscess material cannot be under-estimated. The high yield of positive cultures will then enable the neurosurgeon and the infectious disease specialist to treat brain abscess more rationally and appropriately. Abscesses rarely arise de novo within the brain $[4,11]$. There is almost always a primary lesion elsewhere in the body that must be sought assiduously, since failure to treat the primary lesion will result in relapse [11]. The need to sample the primary source of the brain abscess, especially lungs, when unusual pathogens are isolated from the brain abscess (even in cryptogenic abscesses), is emphasized through our series.

It is advisable for the neurosurgeon to co-ordinate closely with the microbiologist and ensure application of advanced microbiological and molecular techniques, to detect the new and emerging agents of intracranial abscesses.

\section{References}

[1] S. Menon, R. Bharadwaj, A. Chowdhary, D. V. Kaundinya, and D. A. Palande, "Current epidemiology of intracranial abscesses: a prospective 5 year study," Journal of Medical Microbiology, vol. 57, no. 10, pp. 1259-1268, 2008.

[2] C. Sundaram and V. Lakshmi, "Pathogenesis and pathology of brain abscess," Indian Journal of Pathology and Microbiology, vol. 49, no. 3, pp. 317-324, 2006.

[3] E. T. Lisa and N. G. Joshua, "Brain Abscess," eMedicine, Edward Bessman et al., Eds., Medscape, september 2008.

[4] B. Itzhak, "Brain abscess," eMedicine, A. C. Burke et al., Eds., Medscape, June 2008, http://emedicine.medscape.com/ article/212946-overview.

[5] P. H. Lai, J. T. Ho, W. L. Chen et al., "Brain abscess and necrotic brain tumor: discrimination with proton MR spectroscopy and diffusion-weighted imaging," American Journal of Neuroradiology, vol. 23, no. 8, pp. 1369-1377, 2002.

[6] G. E. Mathisen and J. P. Johnson, "Brain abscess," Clinical Infectious Diseases, vol. 25, no. 4, pp. 763-779, 1997.

[7] K. N. Prasad, A. M. Mishra, D. Gupta, N. Husain, M. Husain, and R. K. Gupta, "Analysis of microbial etiology and mortality in patients with brain abscess," Journal of Infection, vol. 53, no. 4, pp. 221-227, 2006.

[8] M. L. Babu, S. K. Bhasin, and Kanchan, "Pyogenic brain abscess and its management," JK Science, vol. 4, no. 1, pp. 2123, 2002.

[9] R. K. Moorthy and V. Rajshekhar, "Management of brain abscess: an overview," Neurosurgical Focus, vol. 24, no. 6, pp. $1-6,2008$.

[10] T. Hakan, "Management of bacterial brain abscesses," Neurosurgical Focus, vol. 24, no. 6, article E4, 2008.

[11] A. R. Tunkel, "Brain abscess, section H, central nervous system infections," in Principles and practice of Infectious diseases, G. L. Mandell, R. G. Douglas, and J. E. Bennett, Eds., vol. 2, pp. 1150-1163, Elsevier, New York, NY, USA, 6th edition, 2005.

[12] V. Lakshmi, R. R. Rao, and I. Dinakar, "Bacteriology of brain abscess-observations on 50 cases," Journal of Medical Microbiology, vol. 38, no. 3, pp. 187-190, 1993.

[13] P. Mary, I. M. James, N. M. Ronald, R. M. Michael, and S. G. Lynne, "Aerobic and anaerobic bacteriology, mycobacteriology, aerobic actinomycetes, antimicrobial susceptibility testing, mycology and parasitology," in Clinical Microbiology Procedures Hand Book, D. I. Henry, Ed., ASM publication, New York, NY, USA, 1992. 
[14] K. Bommakanti, P. Ankathi, P. Uma, S. Malladi, and V. Laxmi, "Cerebral abscess and calvarial osteomyelitis due to Burkholderia pseudomallei," Neurology India, vol. 58, no. 5, pp. 801-802, 2010.

[15] B. Suchanda, A. Rajesh, A. K. Purohit, V. Lakshmi, and C. Sundaram, "A rare concomitant tuberculous and Fonsecaea pedrosoi fungal infection of the skull base," Journal of Neurosciences in Rural Practice. In press.

[16] V. Lakshmi, C. Sundaram, A. K. Meena, and J. M. K. Murthy, "Primary cutaneous nocardiosis with epidural abscess caused by Nocardia brasiliensis-a case report," Neurology India, vol. 50, no. 1, pp. 90-92, 2002.

[17] V. Lakshmi, C. Padmasri, P. Umabala, C. Sundaram, and M. Panigrahi, "Cerebral phaeohyphomycosis due to Cladophialophora bantiana," Indian Journal of Medical Microbiology, vol. 26, no. 4, pp. 392-395, 2008.

[18] C. Sundaram, B. C. Prasad, G. Bhaskar, V. Lakshmi, and J. M. Murthy, "Brain abscess due to Entamoeba histolytica," Journal of Association of Physicians of India, vol. 52, pp. 251-252, 2004.

[19] R. Reddy, M. Vijayasaradhi, M. S. Uppin, S. Challa, A. Jabeen, and R. Borghain, "Acanthamoeba meningoencephalitis in an immunocompetent patient: an autopsy case report," $\mathrm{Neu}$ ropathology, vol. 31, no. 2, pp. 183-187, 2011.

[20] N. Husain, S. Sharma, R. Verma et al., "Multimodal diagnostic approach to brain abscess," The Internet Journal of Tropical Medicine, vol. 3, no. 2, 2007.

[21] J. Carpenter, S. Stapleton, and R. Holliman, "Retrospective analysis of 49 cases of brain abscess and review of the literature," European Journal of Clinical Microbiology and Infectious Diseases, vol. 26, no. 1, pp. 1-11, 2007.

[22] R. Sharma, D. K. Mohan, and R. P. Cooke, "Intracranial abscesses: changes in epidemiology and management over five decades in merseyside," Infection, vol. 37, no. 1, pp. 39-43, 2009.

[23] D. Singh, V. Gupta, A. K. Singh, and S. Sinha, "Evolution of otogenic brain abscess and management protocol," Indian Pediatrics, vol. 38, no. 2, pp. 169-173, 2001.

[24] S. Tandon, N. Beasley, and A. C. Swift, "Changing trends in intracranial abscesses secondary to ear and sinus disease," Journal of Laryngology and Otology, vol. 123, no. 3, pp. 283288, 2009.

[25] A. A. Mueller, B. Saldamli, S. Stübinger et al., "Oral bacterial cultures in non-traumatic brain abscesses: results of a firstline study," Oral Surg Oral Med Oral Pathol Oral Radiol Endod, vol. 107, no. 4, pp. 469-476, 2009.

[26] M. Kagawa, M. Takashita, S. Yato, and K. Kitamura, "Brain abscess in congenital cyanotic heart disease," Journal of Neurosurgery, vol. 58, no. 6, pp. 913-917, 1983.

[27] B. Itzhak, "Microbiology and management of brain abscess in children," Journal of Pediatric Neurology, vol. 2, no. 3, pp. 125130, 2004.

[28] H. Seok, J. L. Dong, G. C. Yong et al., “The multiple brain abscesses associated with congenital pulmonary arteriovenous malformations: a case report," Journal of Korean Medical Science, vol. 17, no. 3, pp. 407-411, 2002.

[29] T. P. Levin, D. E. Baty, T. Fekete, A. L. Truant, and B. Suh, "Cladophialophora bantiana brain abscess in a solid-organ transplant recipient: case report and review of the literature," Journal of Clinical Microbiology, vol. 42, no. 9, pp. 4374-4378, 2004.

[30] S. R. Jayakeerthi, M. Dias, S. Nagarathna, B. Anandh, A. Mahadevan, and A. Chandramuki, "Brain abscess due to
Cladophialophora bantiana," Indian Journal of Medical Microbiology, vol. 22, no. 3, pp. 193-195, 2004.

[31] S.A. Borkar, M. S. Sharma, G. Rajpal, M. Jain, I. Xess, and B. Sharma, "Brain abscess caused by Cladophialophora bantiana in an immunocompetent host: need for a novel cost-effective antifungal agent," Indian Journal of Medical Microbiology, vol. 26, no. 3, pp. 271-274, 2008.

[32] T. Hakan, N. Ceran, I. Erdem, M. Z. Berkman, and P. Gökta, "Bacterial brain abscesses: an evaluation of 96 cases," Journal of Infection, vol. 52, no. 5, pp. 359-366, 2006.

[33] B. Yichayaou, J. Y. Streifler, N. Eynan, and J. Grinblat, "Brain abscess complicating cerebral infarct," Age and Ageing, vol. 31, no. 6, pp. 477-480, 2002.

[34] M. Dias, M. Nagarathna, A. Mahadevan, M. Chandramoulii, and A. Chandramukhi, "Nocardial brain abscess in an immunocompetent host," Indian Journal of Medical Microbiology, vol. 26, no. 3, pp. 274-277, 2008.

[35] G. Barnaud, C. Deschamps, V. Manceron et al., "Brain abscess caused by Nocardia cyriacigeorgica in a patient with human immunodeficiency virus infection," Journal of Clinical Microbiology, vol. 43, no. 9, pp. 4895-4897, 2005.

[36] H. Honda and D. K. Warren, "Central nervous system infections: meningitis and brain abscess," Infectious Disease Clinics of North America, vol. 23, no. 3, pp. 609-623, 2009.

[37] R. N. Khouzam, A. M. El-Dokla, and D. L. Menkes, "Undiagnosed patent foramen ovale presenting as a cryptogenic brain abscess: case report and review of the literature," Heart and Lung, vol. 35, no. 2, pp. 108-111, 2006.

[38] V. Lakshmi, "Culture of body fluids using bacT/Alert system," Indian Journal of Medical Microbiology, vol. 19, no. 2, pp. 6772, 2001

[39] P. T. Kao, H. K. Tseng, C. P. Liu, S. C. Su, and C. M. Lee, "Brain abscess: clinical analysis of 53 cases," Journal of Microbiology, Immunology and Infection, vol. 36, no. 2, pp. 129-136, 2003.

[40] D. Blázquez, M. Muñoz, C. Gil, J. L. Ruibal, F. E. Knaichi, and E. Aleo, "Brain abscess and epidural empyema caused by Salmonella enteritidis in a child: successful treatment with ciprofloxacin: a case report," Cases Journal, vol. 2, no. 6, article 7131, 2009.

[41] A. Chandramuki, A. S. Hegde, and N. Reddy, "Anaerobic brain abscess - role of metronidazole in chemotherapy," Neurology India, vol. 28, pp. 213-218, 1980.

[42] R. Bhardwaj and B. N. Joshi, "Brain-abscesses-a study of the causative organisms with special reference to anaerobes," Indian Journal of Pathology and Microbiology, vol. 31, no. 2, pp. 44-48, 1988.

[43] R. Chaudhry, B. Dhawan, B. V. J. Laxmi, and V. S. Mehta, "The microbial spectrum of brain abscess with special reference to anaerobic bacteria," British Journal of Neurosurgery, vol. 12, no. 2, pp. 127-130, 1998.

[44] S. L. Gorbach, J. W. Mayhew, and J. G. Bartlett, "Rapid diagnosis of anaerobic infections by direct gas liquid chromatography of clinical specimens," The Journal of Clinical Investigation, vol. 57, no. 2, pp. 478-484, 1976.

[45] A. B. Onderdon, S. Myron et al., "Gas-liquid and high performance liquid chromatographic methods for the identification of microorganisms," in Diagnsotic Technologies in Clinical Microbiology, pp. 123-130.

[46] S. S. Shuangshoti and S. Shuangshoti, "Tuberculous brain abscess: a case report with a review of the literature in English," Neuropathology, vol. 19, no. 3, pp. 328-335, 1999.

[47] J. E. Vidal, A. C. Oliveira, R. F. Dauar et al., "Cerebral tuberculomas or tuberculous brain abscess: the dilemma continues," Clinical Infectious Diseases, vol. 40, no. 7, pp. 1072-1073, 2005. 
[48] V. B. Dohe, S. K. Deshpande, and R. S. Bharadwaj, "Tubercular brain abscess_-case report," Indian Journal of Tuberculosis, vol. 57, no. 2, pp. 102-103, 2010.

[49] K. Wanjari, V. P. Baradkar, G. Nataraj, and S. Kumar, "A rare case of tubercular cerebellar abscess," Indian Journal of Medical Microbiology, vol. 27, no. 4, pp. 363-365, 2009.

[50] V. Sharma and G. Newton, "Multiple tuberculosis brain abscesses," Indian Journal of Tuberculosis, vol. 39, no. 3, pp. 185186, 1992.

[51] A. Rajesh, A. K. Purohit, and V. Lakshmi, "Calvarial tubercular osteomyelitic abscess-letter to the editor," Indian Journal of Medical Microbiology, vol. 27, no. 4, pp. 380-381, 2009.

[52] R. Schlaberg, R. C. Huard, and P. D. Latta, "Nocardia cyriacigeorgica, an emerging pathogen in the United States," Journal of Clinical Microbiology, vol. 46, no. 1, pp. 265-273, 2008.

[53] J. H. Shin and H. K. Lee, "Nocardial brain abscess in a renal transplant recipient," Clinical Imaging, vol. 27, no. 5, pp. 321324, 2003.

[54] G. Baracco and G. Dickinson, "Pulmonary nocardiosis," Current Infectious Disease Reports, vol. 3, no. 3, pp. 282-292, 2001.

[55] K. J. Kennedy, K. H. C. Chung, F. J. Bowden et al., "A cluster of Nocardial brain abscesses," Surgical Neurology, vol. 68, no. 1, pp. 43-49, 2007.

[56] F. Athanassiadou, A. Tragiannidis, T. Papageorgiou, and A. Velegraki, "Fungal brain abscesses in leukemia," Indian Pediatrics, vol. 43, no. 11, pp. 991-994, 2006.

[57] M. A. Masalma, F. Armougom, W. M. Scheld et al., "The expansion of the microbiological spectrum of brain abscesses with use of multiple 16 S ribosomal DNA Sequencing," Clinical Infectious Diseases, vol. 48, no. 9, pp. 1169-1178, 2009.

[58] Y. W. Tang, G. W. Procop, and D. H. Persing, "Molecular diagnostics of infectious diseases," Clinical Chemistry, vol. 43, no. 11, pp. 2021-2038, 1997.

[59] P. M. Keller, S. K. Rampini, and G. V. Bloemberg, "Detection of a mixed infection in a culture-negative brain abscess by broadspectrum bacterial 16S rRNA gene PCR," Journal of Clinical Microbiology, vol. 48, no. 6, pp. 2250-2252, 2010.

[60] J. E. Clarridge, "Impact of $16 \mathrm{~S}$ rRNA gene sequence analysis for identification of bacteria on clinical microbiology and infectious diseases," Clinical Microbiology Reviews, vol. 17, no. 4, pp. 840-862, 2004.

[61] J. C. Tsai, J. L. Teng, and P. R. Hsuch, "Direct detection of bacterial pathogens in brain abscesses by polymerase chain reaction, amplification and sequencing of partial $16 \mathrm{~S}$ ribosomal deoxyribonucleic acid fragments," Neurosurgery, vol. 55, no. 5, pp. 1154-1162, 2004.

[62] P. C. Woo, S. K. Lau, J. L. Teng, H. Tse, and K. Y. Yuen, "Then and now: use of $16 \mathrm{~S}$ rDNA gene sequencing for bacterial identification and discovery of novel bacteria in clinical microbiology laboratories," Clinical Microbiology and Infection, vol. 14, no. 10, pp. 908-934, 2008.

[63] D. B. Digiulio and D. A. Relman, "Majority rules? Tallying the microbial census in an abscess by means of molecular methods," Clinical Infectious Diseases, vol. 48, no. 9, pp. 11791181, 2009.

[64] P. H. Lai, K. T. Li, S. S. Hsu et al., "Pyogenic brain abscess: findings from in vivo $1.5-\mathrm{T}$ and $11.7-\mathrm{T}$ in vitro proton $\mathrm{MR}$ spectroscopy," American Journal of Neuroradiology, vol. 26, no. 2, pp. 279-288, 2005.

[65] S. Menon, R. Bharadwaj, A. S. Chowdhary, D. N. Kaundinya, and D. A. Palande, "Utility of in vitro proton magnetic resonance spectroscopy in aetiological characterisation of brain abscesses," Indian Journal of Medical Microbiology, vol. 28, no. 4, pp. 348-353, 2010.

[66] S. Menon, R. Bharadwaj, A. S. Chowdhary, D. N. Kaundinya, and D. A. Palande, "Rapid identification of non-sporing anaerobes using nuclear magnetic resonance spectroscopy and an identification strategy," Indian Journal of Medical Microbiology, vol. 25, no. 4, pp. 330-335, 2007. 


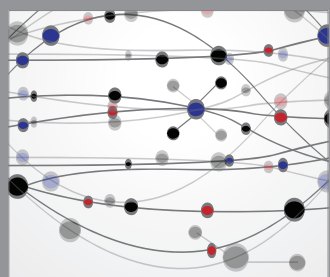

The Scientific World Journal
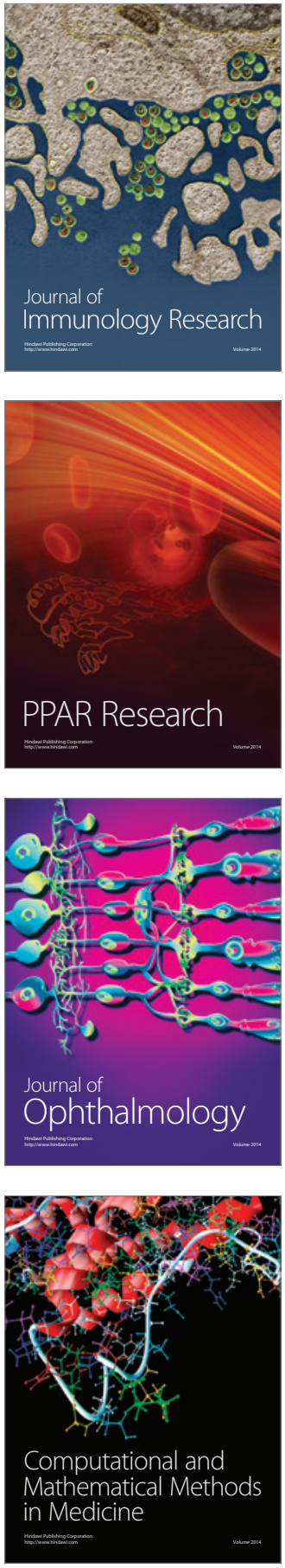

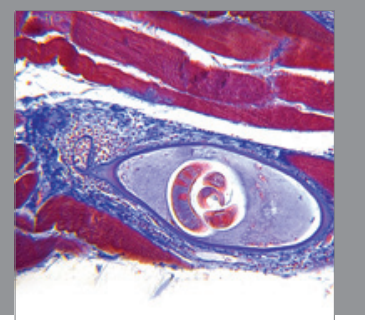

Gastroenterology

Research and Practice
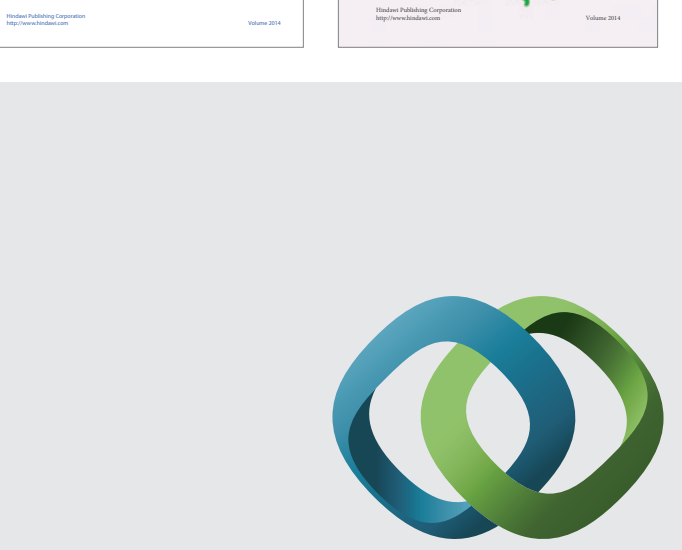

\section{Hindawi}

Submit your manuscripts at

http://www.hindawi.com
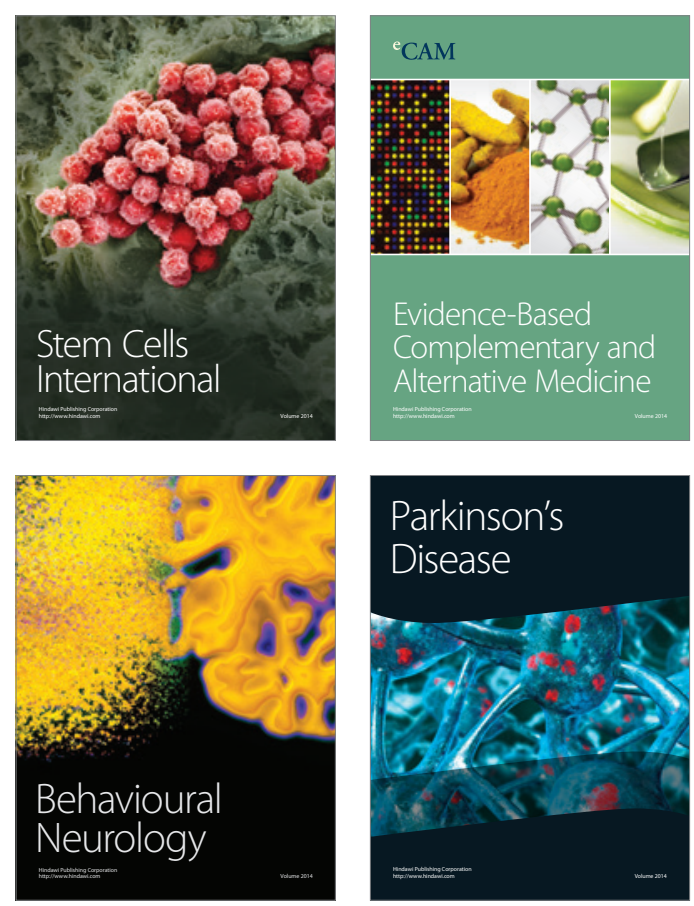

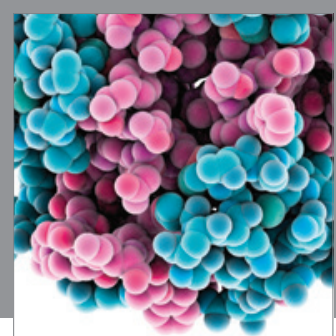

Journal of
Diabetes Research

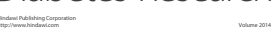

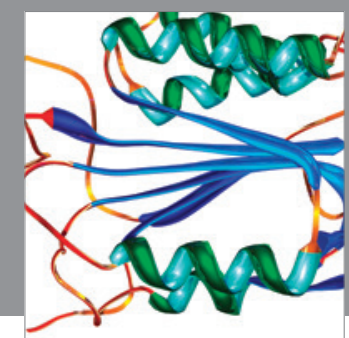

Disease Markers
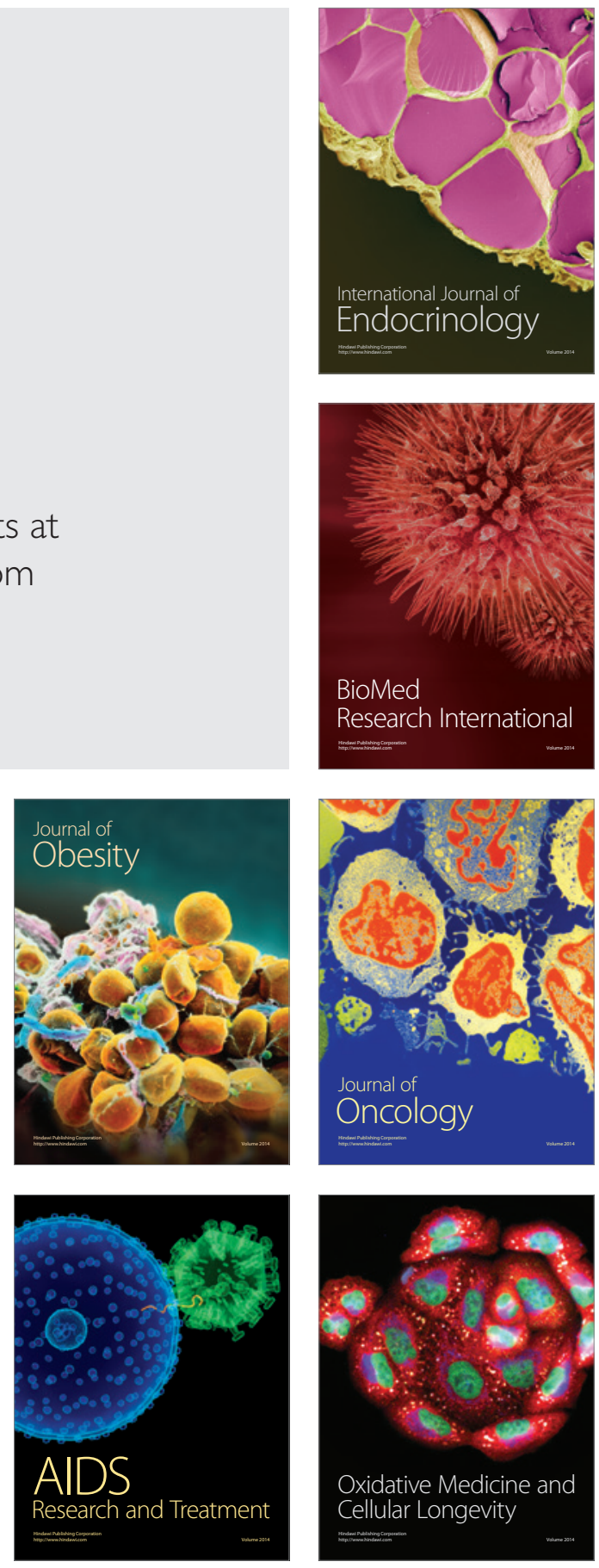